\title{
Response to Comments on "Pharmacokinetics, Pharmacodynamics, and Safety of the Novel Calcimimetic Agent Evocalcet in Healthy Japanese Subjects: First-in-Human Phase I Study"
}

\author{
Tadao Akizawa $^{1} \cdot$ Ryutaro Shimazaki $^{2} \cdot$ Masanari Shiramoto $^{3} \cdot$ Masafumi Fukagawa $^{4} \cdot$ Evocalcet Study Group
}

Published online: 10 January 2019

(c) The Author(s) 2019

We are extremely grateful to Dr. Srinivas for his thoughtful review of our article. His comments about the pharmacodynamic properties of evocalcet were from a clinical development perspective, with a particular focus on the calcium-lowering effect of evocalcet and the resultant risk of corrected QT interval (QTc) prolongation [1].

As described in the letter, while both doses of evocalcet ( 6 and $12 \mathrm{mg}$ ), administered as multiple doses, showed similar efficacy in reducing intact parathyroid hormone (iPTH) levels, the 12-mg dose showed a marked reduction in corrected calcium levels compared with the 6-mg dose in healthy male adults $[1,2]$. Furthermore, both doses showed linear pharmacokinetics in the present study, indicating that the difference between reduction in iPTH and reduction in corrected calcium may depend on the pharmacological effects of evocalcet and/or the physiological responses of subjects to evocalcet [2]. Our results suggest that iPTH may have increased as a biological response to the reduction in corrected calcium after administration of evocalcet in subjects with normal renal and parathyroid function in

This reply refers to the comment available at https://doi. org/10.1007/s40261-018-0738-x.

Tadao Akizawa

akizawa@med.showa-u.ac.jp

1 Division of Nephrology, Department of Medicine, Showa University School of Medicine, Namics 301, 4-24-51 Takanawa, Minato-ku, Tokyo 108-0074, Japan

2 R\&D Division, Kyowa Hakko Kirin Co., Ltd., 1-9-2 Otemachi, Chiyoda-ku, Tokyo 100-0004, Japan

3 SOUSEIKAI Hakata Clinic, Random Square 5-7F, 6-18 Tenyamachi, Hakata-ku, Fukuoka-shi, Fukuoka 812-0025, Japan

4 Division of Nephrology, Endocrinology, and Metabolism, Department of Internal Medicine, Tokai University School of Medicine, 143 Shimokasuya, Isehara-shi, Kanagawa 259-1193, Japan the study, eventually resulting in similar reductions in iPTH levels between the 6 and $12 \mathrm{mg}$ dose groups. This could be attributed to a greater increase in iPTH levels resulting from a larger decrease in corrected calcium at $12 \mathrm{mg}$. In addition, the reduction in iPTH reached a plateau at an iPTH level of approximately $10 \mathrm{pg} / \mathrm{mL}$ at $4 \mathrm{~h}$ after administration at a relatively high dose of 6 or $12 \mathrm{mg}$ in healthy adults with a baseline iPTH level of approximately $30 \mathrm{pg} / \mathrm{mL}$ [2], which is markedly lower than that in hemodialysis patients (e.g. approximately $270 \mathrm{pg} / \mathrm{mL}$ at baseline in a phase $\mathrm{Ib} / \mathrm{II}$ a study in patients with hyperparathyroidism undergoing hemodialysis [3]), thus failing to demonstrate the dose dependence of evocalcet for reduction in iPTH levels. In contrast, the difference in baseline-corrected calcium levels between healthy adults (approximately $9.0 \mathrm{mg} / \mathrm{dL}$ [2]) and patients undergoing hemodialysis (approximately $9.8 \mathrm{mg} / \mathrm{dL}$ in the phase $\mathrm{Ib} /$ IIa study [3]) was not as significant as the difference in $\mathrm{iPTH}$ levels between these groups, successfully demonstrating dose dependence at both 6 and $12 \mathrm{mg}$ for reduction in corrected calcium levels. In fact, the phase Ib/IIa study showed a trend toward dose dependence for reductions in $\mathrm{PTH}$ and corrected calcium at evocalcet doses up to $12 \mathrm{mg}$ in hemodialysis patients, although there was a limitation that the number of patients in the 8 and $12 \mathrm{mg}$ groups was small [3].

Since QTc prolongation is associated with reductions in serum calcium, we are of the opinion that caution should be exercised with the use of evocalcet, as also pointed out by Dr. Srinivas [4-6]. Calcium reduction is one of the characteristic effects of calcimimetics [7, 8], and, indeed, reduction in corrected calcium was correlated with QTc prolongation in our study [2]. Cinacalcet, a currently available calcimimetic, is used worldwide with high efficacy in controlling $\mathrm{iPTH}$, thereby contributing to a reduction in the number of patients undergoing parathyroidectomy [9-12]. Cinacalcet, which also reduces serum calcium, can be successfully used to control iPTH while minimizing the risk of QTc prolongation by adjusting the dose of cinacalcet, along with the dose 
of an active vitamin $\mathrm{D}$ and/or a calcium preparation, taking into account the serum calcium levels [13].

In a phase III study of evocalcet versus cinacalcet, QTc was prolonged after administration in both groups (data not shown in the study by Fukagawa et al. [14]). However, the degree of prolongation was similar between the two groups, and the adverse event of 'QT prolonged', which was reported in two $(2 / 317,0.6 \%)$ and seven $(7 / 317,2.2 \%)$ subjects in the evocalcet and cinacalcet groups, respectively, was mild in all these subjects [14]. In a phase III long-term study of evocalcet (unpublished data), a mild adverse event of 'QT prolonged' was reported in one subject $(1 / 137,0.7 \%)$ during 1-year treatment with dose titration to $12 \mathrm{mg}$. The risk of QTc prolongation associated with the use of evocalcet appeared to be as low as that with cinacalcet. In both these phase III studies, the starting dose was $1 \mathrm{mg}$ in principle and $2 \mathrm{mg}$ for subjects with high iPTH and corrected calcium levels at baseline, followed by an increase in $1 \mathrm{mg}$ increments thereafter, taking into account corrected calcium levels. As a result, a rapid fall in corrected calcium levels and QTc prolongation immediately after administration, which was observed in the high-dose groups in the present study and the phase Ib/IIa study in hemodialysis patients using the fixed doses, were not identified in the phase III studies. In the long-term study, the dose of evocalcet, which was administered once daily, was titrated to as high as $12 \mathrm{mg}$ in 10 subjects (7.3\%). The adverse event of 'QT prolonged' was not reported in subjects treated with $12 \mathrm{mg}$, and all adverse events reported were mild, demonstrating that evocalcet was safely administered.

These findings suggest that evocalcet, like cinacalcet, can be safely used by adjusting the dose to control corrected calcium levels appropriately. In conclusion, it should be emphasized that careful monitoring of QTc prolongation with the use of evocalcet is important, as also indicated by Dr. Srinivas. Furthermore, evocalcet, like cinacalcet, may be expected to control iPTH while reducing safety concerns, including QTc prolongation, by adjusting the dose, taking into account the corrected calcium levels, and by starting treatment at a low dose ( 1 or $2 \mathrm{mg}$ ), followed by a dose increase in increments of $1 \mathrm{mg}$.

\section{Compliance with Ethical Standards}

Funding No funding was received to assist in the preparation of this response.

Conflict of interest TA has received consulting fees from Kyowa Hakko Kirin Co, Ltd, Astellas Pharma Inc., Bayer Yakuhin, Ltd, Fuso Pharmaceutical Industries, Ltd, Japan Tobacco Inc., Ono Pharmaceutical Co, Ltd and NIPRO Industry; as well as lecture fees from Kyowa Hakko Kirin Co., Ltd, Chugai Pharmaceutical Co., Ltd, Bayer Yakuhin, Ltd, Kissei Pharmaceutical Co, Ltd, Torii Pharmaceutical Co, Ltd, and Ono Phar- maceutical Co., Ltd. RS is employed by Kyowa Hakko Kirin Co., Ltd, which funded the original research. MF has received consulting fees from Kyowa Hakko Kirin Co., Ltd and Ono Pharmaceutical Co. Ltd; as well as lecture fees from Kyowa Hakko Kirin Co., Ltd, Bayer Yakuhin, Ltd, Torii Pharmaceutical Co., Ltd and Ono Pharmaceutical Co., Ltd, and grants from Kyowa Hakko Kirin Co., Ltd and Bayer Yakuhin Ltd. MS has no conflicts of interest to declare.

Open Access This article is distributed under the terms of the Creative Commons Attribution-NonCommercial 4.0 International License (http://creativecommons.org/licenses/by-nc/4.0/), which permits any noncommercial use, distribution, and reproduction in any medium, provided you give appropriate credit to the original author(s) and the source, provide a link to the Creative Commons license, and indicate if changes were made.

\section{References}

1. Srinivas NR. Comments on "Pharmacokinetics, pharmacodynamics, and safety of the novel calcimimetic agent evocalcet in healthy Japanese subjects: First-in-Human Phase I study". Clin Drug Investig. 2018. https://doi.org/10.1007/s40261-018-0738-x.

2. Akizawa T, Shimazaki R, Shiramoto M, Fukagawa M, Evocalcet Study Group. Pharmacokinetics, pharmacodynamics, and safety of the novel calcimimetic agent evocalcet in healthy Japanese subjects: first-in-human phase I study. Clin Drug Investig. 2018;38(10):945-54.

3. Shigematsu T, Shimazaki R, Fukagawa M, Akizawa T, Evocalcet Study Group. Pharmacodynamics of evocalcet for secondary hyperparathyroidism in Japanese hemodialysis patients. Clin Exp Nephrol. 2018. https://doi.org/10.1007/s10157-018-1635-6 (Epub 29 Aug 2018).

4. So CS, Batrice L, Volger E. Electrocardiographic changes in electrolyte inbalance. Part 2: alterations in serum calcium [author's transl; in German]. Med Klin. 1975;70(48):1966-8.

5. Beaubien ER, Pylypchuk GB, Akhtar J, Biem HJ. Value of corrected QT interval dispersion in identifying patients initiating dialysis at increased risk of total and cardiovascular mortality. Am J Kidney Dis. 2002;39(4):834-42.

6. Zhang Y, Post WS, Dalal D, et al. Serum 25-hydroxyvitamin D, calcium, phosphorus, and electrocardiographic QT interval duration: findings from NHANES III and ARIC. J Clin Endocrinol Metab. 2011;96(6):1873-82.

7. Borrego-Utiel FJ, Pérez-del Barrio MDP, Biechy-Baldan MDM, Segura-Torres P. Cinacalcet may prolong the QT interval in patients on haemodialysis with secondary hyperparathyroidism. Nefrologia. 2013;33(2):272-3.

8. Temiz G, Yalçın AU, Mutluay R, Bozacı İ, Bal C. Effects of cinacalcet treatment on QT interval in hemodialysis patients. Anatol $\mathbf{J}$ Cardiol. 2016;16(7):520-3.

9. Block GA, Martin KJ, de Francisco AL, et al. Cinacalcet for secondary hyperparathyroidism in patients receiving hemodialysis. $\mathrm{N}$ Engl J Med. 2004;350(15):1516-25.

10. Charytan C, Coburn JW, Chonchol M, et al. Cinacalcet hydrochloride is an effective treatment for secondary hyperparathyroidism in patients with CKD not receiving dialysis. Am J Kidney Dis. 2005;46(1):58-67.

11. Beladi-Mousavi SS, Faramarzi M. Calcimimetic agents in the management of secondary hyperparathyroidism among patients with end-stage renal disease; a review article. J Parathyr Dis. 2015;3(1):12-9.

12. Tentori F, Wang M, Bieber BA, et al. Recent changes in therapeutic approaches and association with outcomes among patients with secondary hyperparathyroidism on chronic hemodialysis: the DOPPS study. Clin J Am Soc Nephrol. 2015;10(1):98-109. 
13. Akizawa T, Kido R, Fukagawa M, et al. Decreases in PTH in Japanese hemodialysis patients with secondary hyperparathyroidism: associations with changing practice patterns. Clin J Am Soc Nephrol. 2011;6(9):2280-8
14. Fukagawa M, Shimazaki R, Akizawa T, Evocalcet Study Group. Head-to-head comparison of the new calcimimetic agent evocalcet with cinacalcet in Japanese hemodialysis patients with secondary hyperparathyroidism. Kidney Int. 2018;94(4):818-25. 